\title{
PRIVAÇÃO DE HOSPEDEIRO E ALIMENTAÇÃO SOBRE AS CARACTERÍSTICAS REPRODUTIVAS DE TRICHOGRAMMA ATOPOVIRILIA (HYMENOPTERA: TRICHOGRAMMATIDAE)
}

\author{
HOST AND FEEDING PRIVATION ON THE TRICHOGRAMMA \\ ATOPOVIRILIA (HYMENOPTERA: TRICHOGRAMMATIDAE) \\ REPRODUCTIVE CHARACTERISTICS
}

Ulysses Rodrigues Vianna ${ }^{1 *}$; Dirceu Pratissoli ${ }^{1}$; José Cola Zanuncio ${ }^{2}$; João Rafael De Conte Carvalho de Alencar ${ }^{1}$; Fernando Domingo Zinger ${ }^{1}$

\section{RESUMO}

Comparou-se o efeito de diferentes períodos de tempo sem hospedeiro nas características reprodutivas de Trichogramma atopovirilia (Hymenoptera: Trichogrammatidae) alimentados ou não. Ovos de Anagasta kuehniella (Lepidoptera: Pyralidae) e Sitotroga cerealella (Lepidoptera: Gelechiidae) foram utilizados como hospedeiros, sendo expostos a fêmeas desse parasitóide após 0, 6, 12, 24, 36, 48, 60, 72, 84 ou 96 horas. Fêmeas de T. atopovirilia, alimentadas parasitaram ovos dos hospedeiros até 96 horas sem os mesmos. No entanto, o parasitismo ocorreu até o período de 24 horas em A. kuehniella e 48 horas com S. cerealella quando não alimentados. O parasitismo, a emergência e a razão sexual de T. atopovirilia foram semelhantes em ovos de A. kuehniella, mas com o aumento do período sem esse hospedeiro, decresce ao longo do tempo, com $S$. cerealella de forma semelhante foi observado para aquelas não alimentadas. $\mathrm{O}$ alimento foi o principal fator que afetou no parasitismo de T. atopovirilia.

Palavras chave: Insecta, Hymenoptera, parasitóide, hospedeiro alternativo, controle biológico.

\begin{abstract}
The effect of time different periods was compared without host in the Trichogramma atopovirilia (Hym.: Trichogrammatidae) reproducitive characteristics fed or not. Anagasta kuehniella (Lepidoptera: Pyralidae) and Sitotroga cerealella (Lepidoptera: Gelechiidae) eggs they were used as hosts, being exposed to that parasitoid females after 0, 6, 12, 24, 36, 48, 60, 72, 84 or 96 hours. T. atopovirilia females, fed and sponged the hosts eggs up to 96 hours without the same ones. However, the parasitism happened until the period of 24 hours in A. kuehniella and 48 hours with S. cerealella when not fed. The T. atopovirilia parasitism, the emergency and the sexual reason were similar in A. kuehniella eggs, but with the increase of the period without that host, it decreases along the time, with $\mathrm{S}$. cerealella in a similar way it was observed for those not fed. The food was the principal factor that affected in the T. atopovirilia parasitism.

Key words: Insecta, Hymenoptera, parasitoid, alternative host, biological control.
\end{abstract}

\section{INTRODUÇÃO}

Espécies do gênero Trichogramma são parasitóides de ovos utilizados mundialmente para o controle de pragas, preferencialmente, da ordem Lepidoptera, como Spodoptera frugiperda (Smith) (Lepidoptera: Noctuidae), considerada a principal praga do milho no Brasil. Trichogramma atopovirilia Oatman \& Platner (Hymenoptera: Trichogrammatidae) é freqüente em posturas de S. frugiperda (Bezerra \& Parra, 2003).

Estudos básicos de Trichogramma são fundamentais para se implementar programas de controle biológico com esse inimigo natural. A qualidade nutricional de hospedeiros alternativos é uma prática rotineira de laboratório para o

\footnotetext{
1 Universidade Federal do Espírito Santo, Dep. de Fitotecnia, Centro de Ciências Agrárias, Caixa Postal 16, CEP 29.500-000, Alegre, ES, Brasil. E-mail: ulyssesvianna@insecta.ufv.br

2 Departamento de Biologia Animal, Universidade Federal de Viçosa, 36571-000, Viçosa, Estado de Minas Gerais, Brasil.

* Autor para Correspondência.
} 
controle da qualidade de espécies e/ou linhagens de Trichogramma produzidos (Oliveira et al., 2000; Pratissoli et al., 2004c).

O hospedeiro alternativo e/ou sua ausência podem influenciar positiva ou negativamente as características biológicas de espécies de Trichogramma (Hohmann et al., 2002; Oliveira et al., 2003; Pratissoli et al., 2004b, d). A qualidade e o desempenho de Trichogramma podem ser influenciados, principalmente, por fatores como o hospedeiro alternativo, a temperatura, a espécie a ser controlada no campo, a arquitetura e a fenologia da planta, além da área de exploração de seu hospedeiro, vento e inseticidas (Pratissoli et al., 2003).

O objetivo foi avaliar a taxa de parasitismo, emergência e razão sexual de T. atopovirilia com ovos dos hospedeiros alternativos Anagasta kuehniella (Zeller) (Lepidoptera: Pyralidae) e Sitotroga cerealella Olivier (Lepidoptera: Gelechiidae) em diferentes períodos após a emergência quando alimentados com mel ou não.

\section{MATERIAL E MÉTODOS}

Este trabalho foi realizado no Laboratório de Entomologia do Núcleo de Desenvolvimento Científico e Tecnológico em Manejo Fitossanitário de Pragas e Doenças (NUDEMAFI) do Centro de Ciências Agrárias da Universidade Federal do Espírito Santo (CCA-UFES), em Alegre - ES, onde foram avaliadas a taxa de parasitismo, emergência e razão de $T$. atopovirilia quanto ao efeito da ausência de alimento e dos hospedeiros A. kuehniella e $S$. cerealella em diferentes intervalos de tempo.

A. kuehniella foi criada segundo Parra (1997) com dieta à base de farinha de trigo integral e de milho $(97 \%)$ e levedura de cerveja (3\%). S. cerealella foi criada segundo Hassan (1997) com modificações do laboratório de Entomologia do CCAUFES, em dieta a base de grãos de trigo com $13 \%$ base úmida (b.u.).

T. atopovirilia, foi criado em recipientes de vidro de 14 x $7 \mathrm{~cm}$, com gotículas de mel na parede interna dos mesmos para a alimentação dos adultos do parasitóide e cartelas de cartolina de 2,5 x $8 \mathrm{~cm}$ com ovos de A. kuehniella, inviabilizados pela exposição a lâmpada germicida por 50 minutos, e de S. cerealella colados com goma arábica diluída em água destilada a 5\%. Esses frascos foram lacrados com filme plástico de PVC para evitar a fuga desse parasitóide.
Fêmeas recém emergidas, de T. atopovirilia, foram individualizadas em tubos de vidro do tipo "Duran" (3,5 x 0,5 cm), os quais foram fechados com filme plástico de PVC. Essas fêmeas foram separadas em dois lotes, com um deles recebendo mel e o outro não. As fêmeas de cada lote foram, também, submetidas à ausência do hospedeiro por $0,6,12,24,36,48,60,72,84$ e 96 horas após a emergência de seus adultos. Cada fêmea recebeu uma cartela, de $3,5 \times 0,5 \mathrm{~cm}$ de cartolina azul celeste, com 40 ovos dos hospedeiros, coletados no dia da oferta, sendo o parasitismo permitido por 24 horas. Foram realizadas 15 repetições por hospedeiro, intervalo de tempo e cada lote de T. atopovirilia. $\mathrm{O}$ experimento foi desenvolvido em câmaras climatizadas a $25 \pm 1{ }^{\circ} \mathrm{C}$, umidade relativa de $70 \pm 10 \%$ e fotofase de 14 horas, até a emergência dos descendentes. Foram avaliadas a taxa de parasitismo, emergência e razão sexual de T. atopovirilia e os dados foram submetidos à análise de regressão linear.

\section{RESULTADOS}

Fêmeas alimentadas de T. atopovirilia parasitaram até o tratamento de 96 horas sem os hospedeiros utilizados (Figura 1A), enquanto isso ocorreu, para fêmeas não alimentadas, até o período de 48 com $S$. cerealella e 24 horas com A. kuehniella (Figura 1B).

Fêmeas alimentadas de T. atopovirilia apresentaram diferentes valores de parasitismo nos dois hospedeiros, com tendência de aumento à medida que não receberam ovos de A. kuehniella. Esse parasitismo apresentou elevação até 36 horas sem $S$. cerealella e decréscimo após esse período houve um decréscimo no parasitismo (Figura 1A). Fêmeas de T. atopovirilia, alimentadas ou não, apresentaram parasitismo semelhante em ovos de S. cerealella (Figura 1A e B). A curva de parasitismo de $T$. atopovirilia apresentou uma queda até 12 horas sem $A$. kuehniella e elevou-se até 24 horas (Figura 1B).

A emergência de T. atopovirilia, alimentado apresentou valores crescentes até 36 horas e decréscimo após esse período com ovos de $S$. cerealella. Essa emergência apresentou variação reduzida entre 0 a 96 horas com A. kuehniella (Figura 2A). Fêmeas desse parasitóide, sem alimento apresentaram queda de emergência até 48 horas em ovos de $S$. cerealella. 


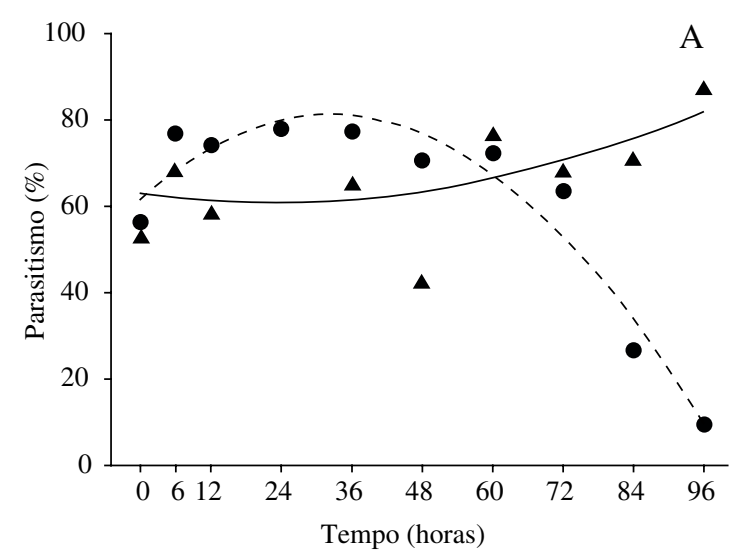

Anagasta kuehniella

$\mathrm{Y}=62.6465-0.169574 \mathrm{x}+0.00385243 \mathrm{x}^{2} \mathrm{R}^{2}=0.55$

Sitotroga cerealella

$\mathrm{Y}=61.7457+1.17654 \mathrm{x}+0.0179478 \mathrm{x}^{2} \mathrm{R}^{2}=0.96$

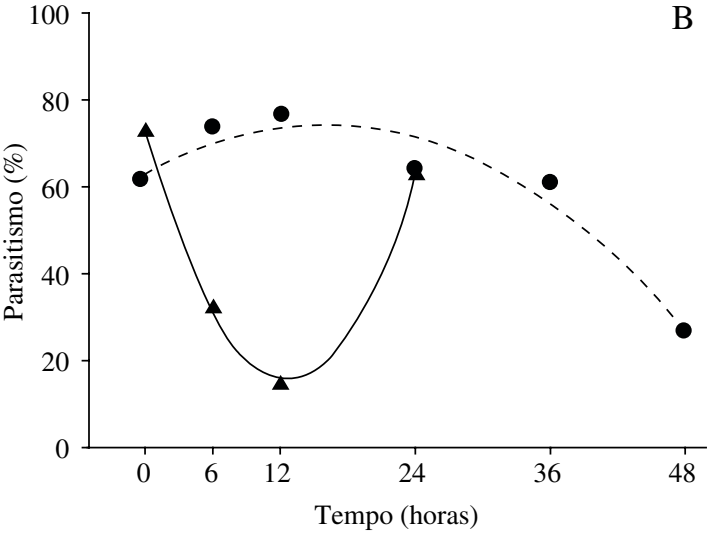

Anagasta kuehniella

$\mathrm{Y}=73.2636-9.18864 \mathrm{x}+0.364773 \mathrm{x}^{2} \mathrm{R}^{2}=0.99$

Sitotroga cerealella

$\mathrm{Y}=62.8656+1.43109 \mathrm{x}-0.044883 \mathrm{x}^{2} \mathrm{R}^{2}=0.96$

\section{A Valores observados A. kuehniella \\ Valores observados $S$. cerealella \\ - Valores estimados A. kuehniella \\ - - - Valores estimados S. cerealella}

Figura 1. Taxa de parasitismo de Trichogramma atopovirilia alimentado (A) ou não (B) com mel por diferentes períodos (horas) sem os hospedeiros Anagasta kuehniella e Sitotroga cerealella. $25 \pm 1{ }^{\circ} \mathrm{C}$; UR $70 \pm 10 \%$ e fotofase de 14 horas.

A
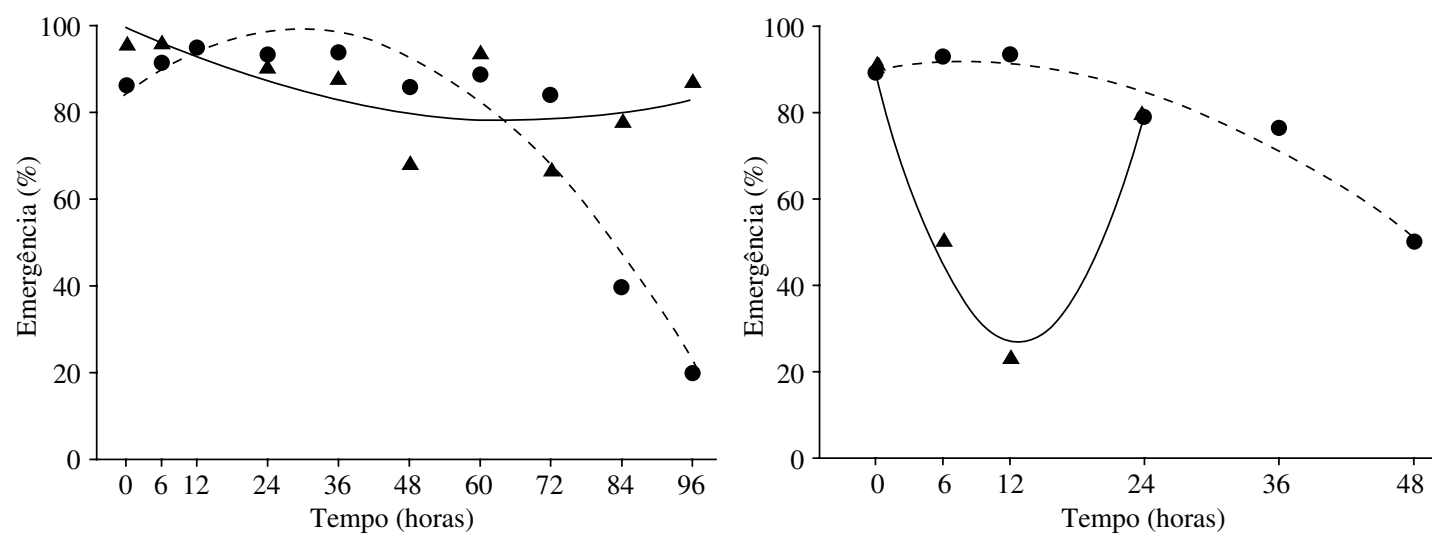

Anagasta kuehniella

$\mathrm{Y}=99.4396-0.634935 \mathrm{x}+0.00477369 \mathrm{x}^{2} \mathrm{R}^{2}=0.69$

Sitotroga cerealella

$\mathrm{Y}=83.9624+1.01635 \mathrm{x}-0.0172408 \mathrm{x}^{2} \mathrm{R}^{2}=0.96$

Anagasta kuehniella

$\mathrm{Y}=92.1364-10.278 \mathrm{x}+0.404672 \mathrm{x}^{2} \mathrm{R}^{2}=0.99$

Sitotroga cerealella

$\mathrm{Y}=90.6072+0.334142 \mathrm{x}-0.0239022 \mathrm{x}^{2} \mathrm{R}^{2}=0.97$ 
A taxa de emergência caiu, drasticamente, até 12 horas sem o hospedeiro $A$. kuehniella e elevou-se até 24 horas (Figura 2B).

A razão sexual de $T$. atopovirilia de fêmeas alimentadas, teve reduzida variação $\operatorname{com} A$. kuehniella. No entanto, a proporção de fêmeas aumentou até 24 horas com $S$. cerealella, e queda após esse período sem esse hospedeiro (Figura 3A). T. atopovirilia, sem alimento, apresentou aumento da razão sexual em ovos de $S$. cerealella até 12 horas sem esse hospedeiro e queda até 48 horas. A razão sexual de $T$. atopovirilia apresentou queda até 12 horas e valores crescentes até 24 horas A. kuehniella (Figura 3B).

\section{DISCUSSÃO}

O parasitismo de fêmeas de T. atopovirilia até 96 horas, quando alimentadas e sem os hospedeiros e até 48 horas para $S$. cerealella e 24 horas para A. kuehniella quando não alimentadas mostrou que a longevidade de Trichogramma depende de alimento e da qualidade do mesmo (Gurr \& Nicol, 2000; Hegazi et al., 2000; Oliveira et al., 2003;
Pratissoli et al., 2004a). Isto mostra que o alimento seja o principal fator que interfere no parasitismo de T. atopovirilia, além do tipo de hospedeiro. O fato do comportamento de parasitismo variar com o hospedeiro utilizado foi relatado também, para seis linhagens de Trichogramma pretiosum Riley em ovos de Tuta absoluta (Meyrick) e Phthorimaea operculella (Zeller) (Lepidoptera: Gelechiidae) e cinco linhagens de $T$. pretiosum em A. kuehniella e S. cerealella (Pratissoli \& Parra, 2001; Pratissoli et al., 2004c).

As maiores taxas de parasitismo de T. atopovirilia alimentadas ou não, em $S$. cerealella ocorreu até 24 horas sem esse hospedeiro, exceto para o período de 0 hora quando essas fêmeas não receberam alimento. S. cerealella seria mais indicado como hospedeiro alternativo para T. atopovirilia, mas isto pode variar entre diferentes linhagens do parasitóide. A linhagem 3 de T. pretiosum apresentou melhores resultados em 12 e 48 horas sem o hospedeiro, enquanto as linhagens 1 e 2 desse parasitóide tiveram maiores taxas de parasitismo de 0 a 48 horas sem o hospedeiro $S$. cerealella, quando suas fêmeas foram alimentadas Pratissoli et al. (2004d). Isto mostra a importância de se avaliar o comportamento de
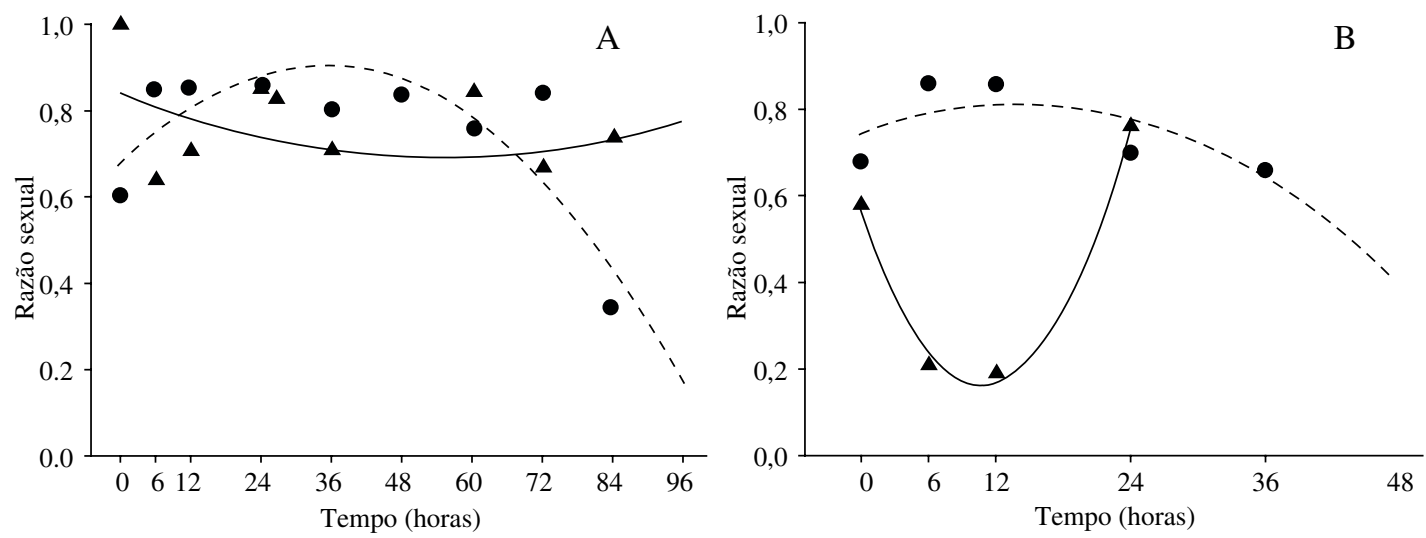

Anagasta kuehniella

$\mathrm{Y}=0.842563-0.00549168 \mathrm{x}+4.99703^{-005} \mathrm{x}^{2} \mathrm{R}^{2}=0.43$

Sitotroga cerealella

$\mathrm{Y}=0.67405+0.0132744 \mathrm{x}-0.000192257 \mathrm{x}^{2} \mathrm{R}^{2}=0.92$

Anagasta kuehniella

$\mathrm{Y}=0.568-0.00751667 \mathrm{x}+0.00347222 \mathrm{x}^{2} \mathrm{R}^{2}=0.99$

Sitotroga cerealella

$\mathrm{Y}=0.740745+0.0104766 \mathrm{x}-0.000368346 \mathrm{x}^{2} \mathrm{R}^{2}=0.94$

\footnotetext{
A Valores observados A. kuehniella

Valores estimados A. kuehniella

- Valores observados $S$. cerealella

- - Valores estimados $S$. cerealella
}

Figura 3. Razão sexual de Trichogramma atopovirilia alimentado (A) ou não (B) com mel por diferentes períodos (horas) sem os hospedeiros Anagasta kuehniella e Sitotroga cerealella. $25 \pm 1{ }^{\circ} \mathrm{C}$; UR $70 \pm 10 \%$ e fotofase de 14 horas. 
seleção hospedeira para diferentes espécies e/ou linhagens de Trichogramma.

A queda na emergência de fêmeas alimentadas de T. atopovirilia a partir de 36 horas sem $S$. cerealella, e a partir de 0 hora com $A$. kuehniella, e taxas de emergência diferentes quando não alimentadas ao longo do tempo sem o hospedeiro A. kuehniella. Isto discorda de resultados de que esses fatos não interferem no percentual de emergência de Trichogramma evanescens Westwood, Trichogramma cacoeciae Marchal e Trichogramma dendrolini Matsumura (Hegazi et al., 2000). Portanto interferência do alimento nas características biológicas de Trichogramma pode variar com a espécie estudada.

O tempo em que $T$. atopovirilia leva para encontrar seu hospedeiro pode interferir no seu percentual de emergência por existir esse decréscimo no parasitismo ao longo da vida do parasitóide alimentados ou não (Reznik et al., 1998; Pratissoli et al., 2004a). Isto pode explicar o comportamento de T. atopovirilia nos dois hospedeiros, pois a tendência de emergência e razão sexual para fêmeas de T. atopovirilia alimentadas ou não foi semelhante à de parasitismo desse parasitóide.

A razão sexual de $T$. atopovirilia, alimentada, apresentou valores maiores que 0,50 nos dois hospedeiros, A. kuehniella e $S$. cerealella, até 72 horas sem os mesmos, o que é considerado uma

\section{LITERATURA CITADA}

BEZERRA, E.B.; PARRA, J.R.P. 2003. Comportamento de parasitismo de Trichogramma atopovirilia Oatman \& Platner e Trichogramma pretiosum Riley (Hymenoptera, Trichogrammatidae) em posturas de Spodoptera frugiperda (J.E. Smith) (Lepidoptera, Noctuidae). Revista Brasileira de Entomologia 47: 205-209.

GURR, G.M.; NICOL, H.I. 2000. Effect of food on longevity of adults of Trichogramma carverae Oatman and Pinto and Trichogramma nr brassicae Bezdenko (Hymenoptera: Trichogrammatidae). Australian Journal of Entomology 39: 185-187.

HASSAN, S.A. 1997. Criação de traça do milho, Sitotroga cerealella, para a produção massal de Trichogramma, In: PARRA., J.R.P.; ZUCCHI, R.A. (Eds.). Trichogramma e o controle biológico aplicado. Piracicaba: FEALQ, p. 183-206.

HEGAZI, E.M; KHAFAGI, W.E.; CORRIGAN, J.E. 2000. Studies on three species of Trichogramma. I. Foraging behaviour for food or hosts. Journal of Applied Entomology 124: $145-149$

HOHMANN, C.L.; LUCK, R.F.; STOUTHAMER, R. 2002. Host deprivation effect on reproduction and survival of Wolbachia-infected and uninfected Trichogramma kaykai característica desejável para a criação massal desse parasitóide (Pak, 1986). Fêmeas não alimentadas de T. atopovirilia apresentaram menor proporção de fêmeas produzidas em 6 e 12 horas sem o hospedeiro A. kuehniella. Isto mostra que a presença de alimento, também, afeta essa característica (Pratissoli et al., 2004a; b; d).

T. atopovirilia necessita de carboidratos e a ausência de alimento interfere na longevidade e fecundidade desse parasitóide. O período sem o hospedeiro também interfere na capacidade reprodutiva desse parasitóide, pois, à medida que as fêmeas ficam sem receber ovos do hospedeiro aumenta a taxa de retenção dos ovos nas fêmeas (Hegazi et al., 2000, Reznik et al., 2001) e afetam a qualidade de sua progênie (Hohmann \& Luck, 2004).

\section{CONCLUSÕES}

1. O alimento é o principal fator que afeta a longevidade de T. atopovirilia;

2. O período sem ovos do hospedeiro aumenta a taxa de retenção de ovos e interfere na qualidade dos descendentes do parasitóide;

3. T. atopovirilia deve ser alimentada e não devem ficar muitas horas sem receber ovos do hospedeiro.

Pinto \& Stouthamer (Hymenoptera: Trichogrammatidae). Neotropical Entomology 30: 601-605.

HOHMANN, C.L.; LUCK, R.F. 2004. Effect of host availability and egg load in Trichogramma platneri Nagarkatti (Hymenoptera: Trichogrammatidae) and its consequences on progeny quality. Brazilian Archives of Biology and Technology 47: 413-422.

OLIVEIRA, H.N.; ZANUNCIO, J.C.; PRATISSOLI, D.; CRUZ, I. 2000. Parasitism rate and viability of Trichogramma maxacalii (Hym.: Trichogrammatidae), parasitoid of the Eucalyptus defoliator Euselasia apisaon (Lep.: Riodinidae), on eggs of Anagasta kuehniella (Lep.: Pyralidae). Forest Ecology and Management 130: 1-6.

OLIVEIRA, H.N.; ZANUNCIO, J.C.; PRATISSOLI, D.; PICANÇO, M.C. 2003. Biological characteristics of Trichogramma maxacalii (Hymenoptera: Trichogrammatidae) on eggs of Anagasta kuehniella (Lepidoptera: Pyralidae). Brazilian Journal of Biology 63: 647-653.

PAK, G.A. 1986. Behavioural variations among strains of Trichogramma spp. Journal Applied of Entomology 101: 55-64.

PARRA, J.R.P. 1997. Técnicas de criação de Anagasta kuehniella, hospedeiro alternativo para produção de Trichogramma, 
In: PARRA., J.R.P.; R.A. ZUCCHI (Eds.). Trichogramma e o controle biológico aplicado. Piracicaba: FEALQ, p. 121-150.

PRATISSOLI, D.; PARRA, J.R.P. 2001. Seleção de linhagens de Trichogramma pretiosum (Hym.: Trichogrammatidae) para o controle das traças Tuta absoluta e Phthorimaea operculella (Lep.: Gelechiidae). Neotropical Entomology 30: 277-282.

PRATISSOLI, D.; VIANNA, U.R.; OLIVEIRA, H.N.; PEREIRA, F.F. 2003. Efeito do armazenamento de ovos de Anagasta kuehniella (Lep.: Pyralidae) nas características biológicas de três espécies de Trichogramma (Hym.: Trichogrammatidae). Revista Ceres 50: 95-103.

PRATISSOLI, D.; OLIVEIRA, H.N.; VIEIRA, S.M.J.; OLIVEIRA, R.C.; ZAGO, H.B. 2004a. Efeito da disponibilidade de hospedeiro e de alimento nas características biológicas de Trichogramma galloi Zucchi (Hymenoptera, Trichogrammatidae). Revista Brasileira de Entomologia 48: 101-104.

PRATISSOLI, D.; HOLTZ, A.M.; GONÇALVES, J.R.; VIANNA, U.R.; BELLINI, L.L. 2004b. Alimentação e ausência de hospedeiro afetando aspectos biológicos de populações de Trichogramma pretiosum. Revista Ceres 51: 587-596.

PRATISSOLI, D.; HOLTZ, A.M.; GONÇALVES, J.R.; OLIVEIRA, R.C.; VIANNA, U.R. 2004c. Características biológicas de linhagens de Trichogramma pretiosum, criados em ovos de Sitotroga cerealella e Anagasta kuehniella. Horticultura Brasileira 22: 396-399.

PRATISSOLI, D.; HOLTZ, A.M.; GONÇALVES, J.R.; VIANNA, U.R.; BELLINI, L.L. 2004d. Efeito da ausência de hospedeiro e de alimento sobre aspectos biológicos de Trichogramma pretiosum Riley (Hymenoptera: Trichogrammatidae). Acta Scientiarum 20: 281-286.

REZNIK, S.Y.; VOINOVICH, N.D.; UMAROVA, T.Y. 1998.

Egg retention in the presence of host in Trichogramma females. Journal of Applied Entomology 122: 555-559.

REZNIK, S.Y.; VOINOVICH, N.D.; UMAROVA, T.Y. 2001. Long-term egg retention and parasitization in Trichogramma principum (Hym., Trichogrammatidae). Journal of Applied Entomology 125: 169-175. 